\title{
Research on the Optimization of Goods Location Based on Greedy Algorithm
}

\author{
Hongmei Ju, ${ }^{1, a}$, Qiqi Liu ${ }^{2, b}$ \\ ${ }^{1}$ School of Beijing Wuzi University, Beijng 101100, China; \\ ${ }^{2}$ School of Beijing Wuzi University, Beijng 101100, China. \\ juhongmei@bwu.edu.cn, boloDaisy@163.com
}

\begin{abstract}
With the rapid development of China's economy, the competition between enterprises is increasing day by day, and it is difficult to form a strong competitive advantage by the advantages of product quality, product type and product cycle. The logistics service for the purpose of cost saving is becoming the world's "Third profit source". In terms of logistics costs, the cost of warehousing and transportation is often the largest. At present, most Chinese enterprises have their own warehouses. Therefore, it is relatively easy to solve the warehouse cost. Reasonable use of the warehouse is also one of the key means to save the cost of inventory.

This article mainly aims at the optimization of the item's position, and chooses the sorting method to optimize the goods for the small batch and multi-frequency items, mainly represented by the auto parts. Based on the general principle of cargo location optimization, the optimal solution of the model is obtained by establishing the mathematical model of cargo location optimization, given the constraint condition, comparing the relevant solution method and combining with the actual needs, and choosing the greedy algorithm to solve the problem. The best program, to achieve cost savings, to achieve the purpose of maximizing the interests of enterprises, has a certain practical significance.
\end{abstract}

Key words: goods location optimization; picking cost; greedy algorithm

\section{Introduction}

Warehousing and transportation as the traditional logistics of the two major priorities, has been the enterprise to improve efficiency, reduce costs in key areas. Therefore, with the development of modern information technology, people are increasingly thinking about how to combine advanced technology and algorithms to rationalize the planning of warehouses and to further optimize the goods location. Aiming at the problem of goods location optimization, many scholars at home and abroad put forward a lot of algorithms, such as ant colony algorithm, genetic algorithm and particle swarm algorithm. Foreign research on goods location optimization is relatively early. Rosenwein proposed a hierarchical storage strategy for goods location [1]; Larson and others used the heuristic algorithm to explore the hierarchical storage strategy based on the Rosenwein research [2]; Sooksaksun and so on based on the classification of storage strategy on the number of roadways, length, product classification and storage to establish a mathematical model, the model to minimize the average walking distance as the goal [3]; Zhan and others studied the multi-level warehouse layout problem with adjacency constraints, established an integer programming model that describes the placement of similar products, and used the tabu search algorithm to solve [4]; In China, Fang He and Yueting Chen considering the goods weight and the stability of the shelf, and optimize the distribution of the cargo based on the improved particle swarm optimization algorithm[5]; and Jian Xiao and Li Zheng considered the relevance of the demand for goods and studied the optimization of the allocation of warehouses [6]; Zhenping Li and Yangtao Yu use the improved single parent genetic algorithm to optimize the warehouse for automated warehouse [7]; Zebo Tan to the location of the goods, the quality of goods and access as a variable to establish the 
location optimization model, but the article on the cargo and other factors lack of consideration [8]; Xizhe Dong and others put forward the article price optimization model based on the turnover rate of good [9]; Jian Wang established a multi-objective optimization model with the dependency of the efficiency of goods entry and exit, the stability of the shelves and the storage of the goods, and the use of the weight coefficient method to convert the multi-objective problem into a single-objective problem[10]; Lingbei Zhu combined with the current characteristics of the domestic market and auto parts, operating efficiency and shelf center as the goal, the auto parts of the automated warehouse for the cargo optimization [11]; Yonghua Du and others have designed a bionic algorithm based on binary particle swarm optimization and genetic algorithm. The experimental results show that the three-dimensional particle group based on the three-dimensional library operation balance and the cargo optimization effect is more significant than the genetic algorithm [12]. In order to solve the problem of goods location optimization, this paper combines the concept of goods location optimization, aims at the sales characteristics of auto parts, introduces the cost factor of picking up and establishes the relevant model. Finally, the greedy algorithm is used to obtain the result, and set a relatively practical solution.

\section{Theory of Goods Location Optimization}

\subsection{The concept of goods location optimization}

The location optimization is based on the current location of the goods based on SKUs and unexpected changes in the dynamic re-allocation of goods in the warehouse location to ensure that the goods are distributed in a more reasonable state, to improve the efficiency of the selection and reduce the warehouse operation The purpose of cost.

There are four storage strategies for location allocation, namely, private storage strategy, classification storage strategy, public storage strategy and random storage strategy. As the public storage, random storage, special storage strategy for the type of goods less, and the requirements of the cargo location is low. So in this article we only consider the classification of storage strategy under the cargo optimization problem.

\subsection{The principle of goods optimization}

Although there are a variety of differences in different distribution centers or warehouses, people will follow some common principles when optimizing the location. This article is mainly to study the shelves and other forms of storage-based multi-channel warehouse, storage products are mainly replacement or consumption of spare parts. The basic characteristics of such goods are: many types, a variety of forms of packaging, out of the library operations are relatively high requirements, first-in first-out operations and so on. Order of such parts often have many frequency, small batch, multi-species characteristics. Due to the high-frequency, small-volume, and such goods on the category management requirements more specific, it is more appropriate to use the following principles to establish an optimization model.

2.2.1. The principle of high efficiency based on turnover

The goods will be sorted by the turnover rate from large to small, and then this order is divided into several sections, belong to a section of the goods listed as the same level, in accordance with the principle of positioning or classification of storage law, specify the storage area to each level of goods, turnover The higher the rate, the closer the entrance is, that is, what is commonly said to distribute the gold area to the goods with high picking frequency.

\subsubsection{Product identity principle}

The principle of identity refers to the principle of keeping the same item in the same place. This allows the operator to be familiar with the location of the goods, and access to the same item to spend the least time to transport, improve the distribution center or warehouse operations efficiency. The principle of identity is usually considered in the constraints.

2.2.3. The principle of light in the top and heavy in the bottom

In the case of goods location assignment, it is necessary to place the heavier goods under the shelves according to the principle of light in the top and heavy in the bottom, while the lighter goods are placed above the shelves. This can keep the shelf center of gravity lower, its stability is 
better.

\section{Establishment of goods location optimization model}

\subsection{Model selection}

In the initial stage of the optimization of the goods, we clearly defined that a commodity corresponds only to one cargo, that is, based on the principle of identity described above. Thus, we can take each shipment to the exit distance value, which is the corresponding picking path value for each item, which can be obtained by actual measurement.

Often in the distribution center or warehouse of the enterprise for the optimization process, there is a very important purpose is to shorten the picking personnel picking path as possible to improve the efficiency of picking. The above three types of optimization principles, mainly from this purpose.

Thus, we can use a simple mathematical model to express. First set a few important parameters:

$x_{i j}$ means 0, 1 variable, when the goods $i$ in the ware location $j$, that is 1 ; when the goods $i$ are not on the ware location $j$, that is 0 .

$L_{I}$ indicates the path distance from the inventory $x_{i}$ position to the exit.

$T_{i}$ that the inventory turnover rate, is the picking frequency.

The ultimate goal of goods optimization in the model is to minimize $\sum_{i=1}^{m} \sum_{j=1}^{n} x_{i j} L_{j} T_{i}$, that is

$$
F=\min \sum_{i=1}^{m} \sum_{j=1}^{n} x_{i j} L_{j} T_{i}
$$

However, the factors that affect the efficiency of picking are not only the picking path, but also the number of picking up the goods, the weight, and the cost of using other equipment in the picking process. In view of this situation, this paper introduces a picking cost coefficient $C$, which expresses the influencing factors other than the path factor, and regards it as picking cost.

The cost of the picking cost $C$ is set or defined according to the actual situation of the enterprise. When collecting the specific data, it can be calculated according to the average quantity of each pick, the weight or volume of the physical goods, and the asset consumption of the picking tool cost, etc. to consider. This paper is mainly from the human cost and mechanical costs to pay two aspects of consideration, the establishment of a function of different weight in the process of picking up the goods to pay the cost of manpower and mechanical use costs. details as follows:

$C$ mainly refers to the labor costs and mechanical costs paid during the picking process, $m$ refers to the weight of the selected goods.

$$
C=\left\{\begin{array}{c}
1, m \leq 15 \\
\lambda(m-15)+1, m>15
\end{array}\right.
$$

This article in the 15kg boundary line is based on the People's Republic of China Technical Supervision issued by the Ministry of Labor of the People's Republic of China to carry out the weight limit (male) selected, with a certain degree of scientific and authoritative. Where $\lambda$ is the coefficient, which can be set according to the historical data of different goods.

In combination with the above, the picking cost coefficient $c$ is added to the optimization model, the goods optimization to achieve the purpose of $\mathrm{F}=\sum_{i=1}^{m} \sum_{j=1}^{n} x_{i j} L_{j} T_{i} C_{i}$ to take the smallest.

\subsection{Constraint condition}

After defining the objective function, we can establish the relevant constraints according to the principle of goods location optimization.

According to the principle of identity, we believe that each ware location storage up to a goods; and each goods can only be stored in a ware location, so the constraints are as follows:

$$
\begin{gathered}
\sum_{j=1}^{n} x_{i j}=1, j=1,2, \cdots, m \\
\sum_{i=1}^{m} x_{i j} \leq 1, i=1,2, \cdots, n \\
x_{i j}=0,1 ; j=1, \cdots, m ; i=1, \cdots, n
\end{gathered}
$$

More scholars choose to "light things on the top and heavy things on the bottom" principle established as the objective function, to obtain the minimum center of gravity. However, 
multi-objective optimization is more complicated in the calculation process, and because of the importance of each principle because of the different degree of empowerment process is difficult to achieve scientific accuracy. Therefore, in this article, we only limit the shelf center of the gravity of the shelf in the lower part to ensure the stability of the shelf. details as follows:

$$
\sum_{i=1}^{m} \sum_{j=1}^{n} x_{i j} h_{j} M_{i} \leq 0
$$

$x_{i j}$ means 0, 1 variable, when the goods $i$ in the cargo $j$, that is 1 ; when the goods $i$ are not on the cargo $j$, that is 0 .

$m_{i}$ is the weight of the goods $i$.

$h_{j}$ is the relative height of the ware location $j$ (to the horizontal axis of the shelf as the horizontal axis, $h_{j}$ is the height relative to the horizontal axis of the coordinate system. For example, the shelf is 6 meters high, 3 meters to the establishment of the horizontal axis of the shelves. Then, when $h$ is 2, the actual meaning of $2_{1}$ is the first ware location at the height of 5 meters. The same is located in the 5 meters high at the second ware location $2_{2}$, the third ware location $2_{3}$ and so on the value of $h$ are 2)

\subsection{Model}

Objective function

$$
F=\min \sum_{i=1}^{m} \sum_{j=1}^{n} x_{i j} L_{j} T_{i} C_{i}
$$

Constraints

$$
\begin{gathered}
\sum_{i=1}^{m} \sum_{j=1}^{n} x_{i j} h_{j} M_{i} \leq 0 \\
\sum_{j=1}^{n} x_{i j}=1, j=1,2, \cdots, m \\
\sum_{i=1}^{m} x_{i j} \leq 1, i=1,2, \cdots, n \\
x_{i j}=0,1 ; j=1, \cdots, m, i=1, \cdots, n
\end{gathered}
$$

The objective function shows that we require the highest efficiency of picking up, that is, the cost of picking is minimized. The constraints show that we always require the shelf center of the gravity to be in the lower part of the shelf during the optimization of the cargo space; we believe that each ware location storage up to a goods; and each goods can only be stored in a ware location. So as to meet the requirements of goods optimization to follow the principle of "light in the top and heavy in the bottom" and "identity".

\section{Algorithm and solving process}

\subsection{Algorithm selection}

In this model, we need to solve $=\min \sum_{i=1}^{m} \sum_{j=1}^{n} x_{i j} L_{j} T_{i} C_{i}$, that is, the optimal solution of the objective function. For the average enterprise, it is not economical to use a very complex or time-consuming algorithm to find a precise optimal solution. Although there are a lot of intelligent algorithms can be exhausted all the process and get the optimal solution of the function, but in the process of solving the cost of a lot of business people and time. There are many algorithms that can be used to solve the problem of goods location optimization, such as genetic algorithm, particle swarm algorithm, etc. Although these algorithms are more efficient, but because of these algorithms are more difficult, longer computing time, so more suitable for large quantities but with high precision requirements of the goods location optimization model. So we in the cargo optimization, should be based on their different needs, the establishment of different models, and need to select a different algorithm to solve.

In this paper, the greedy algorithm is used in the process of solving the problem. The algorithm does not take too much time and manpower. Although it can't get the optimal solution, it can usually get a satisfactory solution, especially for multiple orders, high-frequency, small-volume 
delivery of the distribution center or warehouse to optimize the activities of the warehouse, while the task of distribution tasks assigned to the center or warehouse, in the face of customer "faster" distribution needs, "time" is often Is the most important. Therefore, we aimed at the traditional logistics industry in the specific requirements of the location and based on the improvement of customer service considerations, the use of greedy algorithm to solve.

\subsection{Solving process}

Greedy algorithm is a solution to find the best local solution. It does not pursue the global optimal solution, only hope to get their own more satisfied with the solution. Because it eliminates the amount of time it takes to find the best solution to exhaust all possible, usually based on the current situation, without considering the possible overall situation, but for the whole, get a more satisfactory solution. We in the following specifically wrote the greedy algorithm to solve the steps: Step1: Calculate the total turnover rate of each item of goods, that is, the frequency of picking $T_{i}$; the distance from each shipment to the exit $L_{j}$; and the cost of picking for each category of goods $C_{i}$.

Step2: the goods in accordance with $T_{i} C_{i}$ descending (non-incremental) arrangement number, set $G(g)$ for the value of the sorted sequence, $g$ is the serial number.

Step3: the goods in accordance with $L_{j}$ ascending order number, set $P(p)$ for the value of the sorted sequence, $p$ for the serial number.

Step4: Store the goods with the largest $g$ in the lowest $p$ value. That is, the number 1 in the column $G(g)$ is assigned to the goods in the sequence $P(p)$ number 1 , and then the number 2 of the kind of goods assigned to the $P(p)$ number 2 of the goods location, and this is repeated. Process until all the items in the column $G(g)$ are assigned.

We can see that in the process of solving the greedy algorithm, we do not need a complex programming basis, even without advanced mathematical theory, only a simple algorithm can be found in the objective function of the relative optimal solution. Its calculation is faster and the required manpower and material resources are less, so it is a more economical and more appropriate solution.

\section{Conclusion}

\subsection{Model advantage}

In the process of constructing the cargo position optimization model, the main innovations of this paper are as follows:

During the establishment of the model, a picking cost factor is inserted to describe the associated costs that may arise during the picking process. As the picking process is a complex process, its operation is often difficult to quantify. After the introduction of picking costs, the weight of the items, the required equipment and other factors are taken into account, to a certain extent make the model more complete.

Using the greedy algorithm to solve the process, the greedy algorithm is more computationally faster and easier than the genetic algorithm. Compared with the characteristics of the logistics industry, this paper finds that there is no need for high accuracy in the process of solving the model, so you can make up for the greedy algorithm is not the overall optimal limitations.

\subsection{Other principles and optimization significance}

This paper mainly considers the above three principles in the process of model establishment, but there are some very important principles in the theory of cargo location optimization, such as product relevance principle. The analysis found that some products had the same attributes, placing the relevant products in the same area, which would reduce the path of picking up inventory, reduce staff fatigue, and simplify inventory work. Specifically, the demand for some goods is not independent, such as screws and nuts. Since both often appear in an order at the same time, they are preferably assigned to the same place of goods from the point of view of improving the efficiency of picking. So in the optimization process to consider the relevance of the goods. We usually analyze the historical order to obtain the correlation between the goods, so as to optimize the cargo 
location.

In addition to the above mentioned points, there are many principles need to be considered, such as the principle of first-in first-out, the principle of dividing the roadway, the principle of storage area, the shortest line storage principles, the principle of cargo relevance and so on. This article in the process of establishing the optimization model is not to say that all the turnover rate of large auto parts must be close to the entrance, and the bottom of the shelf is not fully meet the parts storage conditions. The ultimate goal of optimization is to achieve global optimal, so it is required that some individuals may need to compromise to other objectives, in order to achieve the overall optimal purpose.

By continually optimizing the location, companies can improve the efficiency of the picking operations of the distribution center or warehouse, and can make the relevant operating costs more rationalized, the most important thing is to enable the company's distribution behavior faster and more convenient, so as to improve customer satisfaction with the enterprise, enhance the profitability of enterprises. Therefore, a reasonable layout of the goods in reducing logistics costs, improve logistics efficiency and customer service capabilities have played a very important role.

\section{Acknowledgments}

This paper is supported by Beijing Key Laboratory (NO: BZ0211), Beijing Intelligent Logistics System Collaborative Innovation Center and Beijing Institute of Materials Research support funding.

\section{References}

[1] Rosenwein M B. An application of cluster analysis to the problem of locating items within a warehouse [J]. IIE Transcations, 1994,26 (1): 101-103.

[2] Larson T N, March H, Kusiak A. A heuristic approach to warehouse layout with class based storage [J]. IIE Transactions, 1997,1 (29): 337-348.

[3] Roodbergen K J, Vis I F A. A model for warehouse layout [J]. IIE Transactions, 2006, 38 (10): 799-811.

[4] Zhang G Q, Lai K K. Tabu search approaches for the multi-level warehouse layout problem with adjacency constraints [J]. Engineering Optimization, 2010,42 (8): 775-790.

[5] Yueting Chen, Fang He. Optimization of Freight Warehouse Based on Genetic Algorithm [J]. Logistics Technology, 2008, (1): 38-41.

[6] Zhenping Li, Yangtao Yu. Application of side-by-side selection of single parent genetic algorithm in optimization of warehouse location in automatic warehouse [J]. Mathematics in Practice and Cognition, 2016: 179-186.

[7] Zhishuai Liu, Lingyun Tong, Lipeng Wei, Kai Zhu, Xiaoli Qian. Design of cargo location based on greedy algorithm [J]. Logistics Technology, 2013, (9): 99-101.

[8] Zebo Tan. Three-dimensional warehouse location selection optimization model [J]. Center Enterprise Science and Technology, 2007 (10): 56-58.

[9] Xizhe Dong. Research on Optimization Model and Algorithm of Storage and Delivery [J]. Harbin: Harbin Institute of Technology, 2004.

[10] Jian Wang. Based on the genetic algorithm of storage and storage optimization [D]. Xi'an: Xi'an University of Architecture and Technology, 2009.

[11] Lingbei Zhu. Auto parts storage characteristics and automated storage location optimization [J]. Management Manager, 2017 (2): 395-397.

[12] Yonghua Du, Qian Xiang, Zhijun Lv, Dong Chen.Study on Biomass Algorithm of Stereo Library Operation Equilibrium and Batch Optimization [J] .2017 (1): 122-126.

[13] Karoon Suksonghong, Kittipong Boonlong, Kim-Leng Goh. Multi-objective genetic algorithms for solving clarion optimization problems in the electricity market [J]. Electrical Power and Energy Systems, 2014, 58: 150-159. 